\title{
Description of Venous Thromboembolism in Hospitalized Patients With Metastatic Cancer: A National Sample
}

\author{
Kahee A. Mohammed, MD, MPH,a,b; Leslie Hinyard, PhD, MSW' ${ }^{\mathrm{b}}$; Martin W. Schoen, MD, MPH'; \\ Christian J. Geneus, MS, MPH'd Eric S. Armbrecht, PhD $^{\text {b }}$; Fred R. Buckhold, MD ${ }^{a}$; and \\ Thomas E. Burroughs, $\mathrm{PhD}^{\mathrm{b}}$
}

\begin{abstract}
Background: This study aimed to determine patient-, tumor-, and hospital-level characteristics associated with venous thromboembolism (VTE), and to assess the impact of VTE on in-hospital mortality and length of hospital stay in hospitalized patients with metastatic cancer. Methods: Using the Nationwide Inpatient Sample database, a cross-sectional analysis was performed of patients aged $\geq 18$ years with at least 1 diagnosis of primary solid tumor and subsequent secondary or metastatic tumor between 2008 and 2013. Results: Among 850,570 patients with metastatic cancer, $6.6 \%$ were diagnosed with VTE. A significant trend for increasing VTE rates were observed from 2008 to 2013 (5.7\%-7.2\%; $P<.0001)$. Using an adjusted multilevel hierarchical regression model, higher odds of VTE were seen among women (odds ratio [OR], 1.04; $95 \% \mathrm{Cl}, 1.02-1.06)$, black versus white patients $(\mathrm{OR}, 1.14 ; 95 \% \mathrm{Cl}, 1.11-1.18)$, and those with an Elixhauser comorbidity index score of $\geq 3(\mathrm{OR}, 2.50 ; 95 \% \mathrm{Cl}, 2.38-2.63)$. Hospital-level correlates of VTE included treatment in a teaching hospital (OR, 1.05; $95 \%$ $\mathrm{Cl}, 1.01-1.11)$ and an urban location (OR, 1.18; $95 \% \mathrm{Cl}, 1.09-1.27)$, and admission to hospitals in the Northeast (OR, 1.16; 95\% $\mathrm{Cl}, 1.08-1.24)$ and West (OR, 1.09; $95 \% \mathrm{Cl}, 1.03-1.16)$ versus the South. Patients with metastasis to the liver, brain, or respiratory organs and those with multiple $(\geq 2)$ metastatic sites had higher odds of VTE, whereas those with metastasis to lymph nodes and genital organs had lower odds. Patients diagnosed with versus without VTE had higher odds of in-hospital mortality $(\mathrm{OR}, 1.50 ; 95 \% \mathrm{Cl}, 1.38-1.63)$ and prolonged hospital stay $(\mathrm{OR}, 1.65 ; 95 \% \mathrm{Cl}, 1.57-1.73)$. Conclusions: The frequency of VTE in patients with metastatic cancer is increasing. Patient characteristics, hospital factors, and site of metastasis independently predict the occurrence of VTE and allow for better stratification of patients with cancer according to their VTE risk.
\end{abstract}

Venous thromboembolism (VTE) is a leading cause of morbidity and mortality in patients with cancer. ${ }^{1,2} \mathrm{~Pa}$ tients with cancer and VTE have a lower survival rate; Sorensen et $\mathrm{al}^{1}$ reported a 1 -year survival rate of $12 \%$ in patients with VTE compared with $36 \%$ of those without. Additionally, patients with cancer and VTE are at a 4-to 8-fold higher risk of death relative to those without cancer. ${ }^{3,4}$ During hospitalization, patients with

From the a Department of Internal Medicine, School of Medicine, Saint Louis University, 'bSaint Louis University Center for Health Outcomes Research (SLUCOR), and 'Division of Hematology/Oncology, Department of Medicine, Saint Louis University School of Medicine, St. Louis, Missouri; and dDepartment of Biostatistics and Bioinformatics, School of Public Health and Tropical Medicine, Tulane University, New Orleans, Louisiana. Submitted February 23, 2017; accepted for publication September 13, 2017

The authors have disclosed that they have no financial interests,
VTE have higher rates of in-hospital mortality and a prolonged hospital stay compared with those without VTE. ${ }^{5,6}$ The economic burden of VTE in United States is enormous, with an estimated annual cost of $\$ 7$ to $\$ 10$ billion USD each year in medical care. ${ }^{7}$ Previous studies have reported varied incidence of VTE in hospitalized patients with cancer, ranging from $0.6 \%$ to $7.8 \% .5,8,9$ This inconsistency is likely due to heterogeneity of the

arrangements, affiliations, or commercial interests with the manufacturers of any products discussed in this article or their competitors.

Author contributions: Study concept and design: Mohammed, Armbrecht, Buckhold, Burroughs. Data acquisition: Mohammed, Geneus. Data analysis and interpretation: Mohammed, Hinyard, Geneus. Manuscript preparation: all authors. Critical revision: all authors.

Correspondence: Kahee A. Mohammed, MD, MPH, Department of Internal Medicine, School of Medicine, Saint Louis University, 3635 Vista Avenue, FDT 14th Floor, St. Louis, MO 63104. E-mail: kmohamm2@slu.edu 
cancer, sampling variability, and variation in VTE surveillance and outcome measures. ${ }^{10-13}$ Major risk factors for the development of VTE include advanced age, major surgery, obesity, medical comorbidities, and immobilization. ${ }^{14,15}$ Risk factors for VTE in patients with cancer include the primary tumor site, receipt of chemotherapy, and presence of metastatic disease. ${ }^{8,9,16,17}$

Patients with metastatic cancer are now living longer. However, factors such as increased hypercoagulability, larger tumor burden, and impaired physical activity place these patients at increased risk of preventable disease, such as VTE. ${ }^{18,19}$ The distribution, correlates, and short-term outcomes associated with VTE in hospitalized patients with metastatic cancer are not well-defined. This study aimed to (1) describe the prevalence of VTE among hospitalized patients with metastatic cancer; (2) examine the association between patient-, tumor-, and hospitallevel characteristics and the occurrence of VTE; and (3) assess the impact of VTE on in-hospital mortality and hospital length of stay (LOS) among patients with metastatic cancer.

\section{Methods}

\section{Data Source}

This study involved cross-sectional analysis of hospital discharge data from the Nationwide Inpatient Sample (NIS) database between 2008 and 2013. The NIS data are obtained from the Healthcare Cost and Utilization Project (HCUP) of the Agency for Healthcare Research and Quality (AHRQ). ${ }^{20}$ It is the largest publically available all-payer inpatient care database in the United States containing data on $>7$ million hospital stays each year (estimating $>30$ million weighted hospitalizations nationally), representing $20 \%$ of a stratified random sample of all hospital discharges from approximately 1,050 hospitals across 44 states. Detailed information on the NIS design and sampling methods are described elsewhere. ${ }^{20}$ NIS is a publically available database containing deidentified patient information and was reviewed as an exempt project by the Saint Louis University Institutional Review Board.

\section{Patient Population}

Patients aged $\geq 18$ years with at least 1 diagnosis of a primary solid tumor were identified using ICD-9-CM diagnostic codes (supplemental eTable 1, available with this article at JNCCN.org). Using secondary diagnostic codes, only patients with metastasis were included in the study.

\section{Outcome Variables}

The primary outcome of interest was the presence of VTE among hospitalized patients diagnosed with metastatic cancer. VTE was defined as pulmonary embolism (PE) and/or deep vein thrombosis using relevant ICD-9 diagnosis codes as described by AHRQ Patient Safety Indicators (supplemental eTable 1). ${ }^{21}$ These codes have been validated to be appropriate for the identification of VTE using administrative data. ${ }^{22}$ Patients with superficial thrombophlebitis and thrombosis were excluded, as were those with chronic VTE.

Secondary outcome measures were in-hospital mortality and hospital LOS. In-hospital mortality was defined as death that occurred during hospitalization, as coded from the discharge disposition of the patient. Hospital LOS was dichotomized into prolonged hospital stay (yes/no) if the LOS was $\geq 75$ th percentile based on the presence or absence of a major operating room procedure related to the same admission for each patient. ${ }^{23}$ These adverse outcomes were selected based on their previously described association with VTE. ${ }^{6}$

\section{Patient and Hospital Characteristics}

Sociodemographic variables examined included sex, age, race/ethnicity (white, black, Hispanic, other), and health insurance status. These variables were selected based on their association with VTE, as documented in previous studies., ${ }^{5,11}$ Comorbidities were classified using the Elixhauser comorbidity index ${ }^{24}$ and the final variable was a summation of the number of comorbid conditions defined by the index. Because previous studies have indicated that patients with cancer undergoing major surgery or an oncologic procedure are at increased risk for VTE, ${ }^{25,26}$ we assessed a major operating room procedure (yes/no) related to the same admission. This variable was predefined in the NIS database using ICD-9-CM procedure code for any major diagnostic or therapeutic operating room procedure. Hospital-level characteristics included in this analysis were hospital location (rural vs urban), hospital teaching status (nonteaching vs teaching), hospital size (small, medium, large), 
and geographic location of the hospital (Northeast, Midwest, South, West).

\section{Statistical Analysis}

Available patient sociodemographic, clinical, and hospital-level characteristics were compared between patients with and without VTE using $\chi^{2}$ or $t$ test, as appropriate. Trends in the prevalence of VTE and in-hospital mortality rates in patients with and without VTE were assessed using the Cochran-Armitage test. Multilevel hierarchical logistic regression using generalized linear mixed models with generalized estimated equations were used to examine the association between patient, tumor, and hospital characteristics and study outcomes. Variables that were significant on univariate regression were included in the multivariable models. We required hospitals have at least 10 patients diagnosed with metastatic cancer for inclusion in the analysis to appropriately measure hospital-level variability. Data distribution and analyses were weighted according to the standard NIS approach to account for the complex sampling design and provide nationally representative estimates. All tests were 2 -sided with an a priori $\alpha$ of 0.05. All analyses were performed using SAS version 9.4 (SAS Institute Inc., Cary, North Carolina).

\section{Results}

\section{Baseline Characteristics}

Characteristics of 850,570 patients aged $\geq 18$ years with a diagnosis of metastatic cancer stratified by the presence or absence of VTE are described in Table 1. Approximately $6.6 \%$ of patients had a diagnosis of $\operatorname{VTE}(\mathrm{n}=56,302)$. Most of the patients were women $(51.3 \%)$, were $\geq 65$ years of age $(53.0 \%)$, were white (72.1\%), had health insurance $(94.0 \%)$, had a major operating room procedure related to the same admission $(30.0 \%)$, were admitted to a teaching hospital $(53.4 \%)$, and were admitted to an urban hospital (90.0\%).

Weighted distribution of primary and metastatic tumor sites in the study sample is depicted in Table 2. Most of the patients had lung cancer $(29.2 \%)$ as the primary tumor site, followed by colorectal (16.2\%), breast $(11.8 \%)$, prostate $(7.8 \%)$, and pancreatic (7.1\%) cancers. Approximately $2.3 \%$ had multiple primary cancers. The most common sites of metastasis were liver $(28.4 \%)$, bone and bone marrow

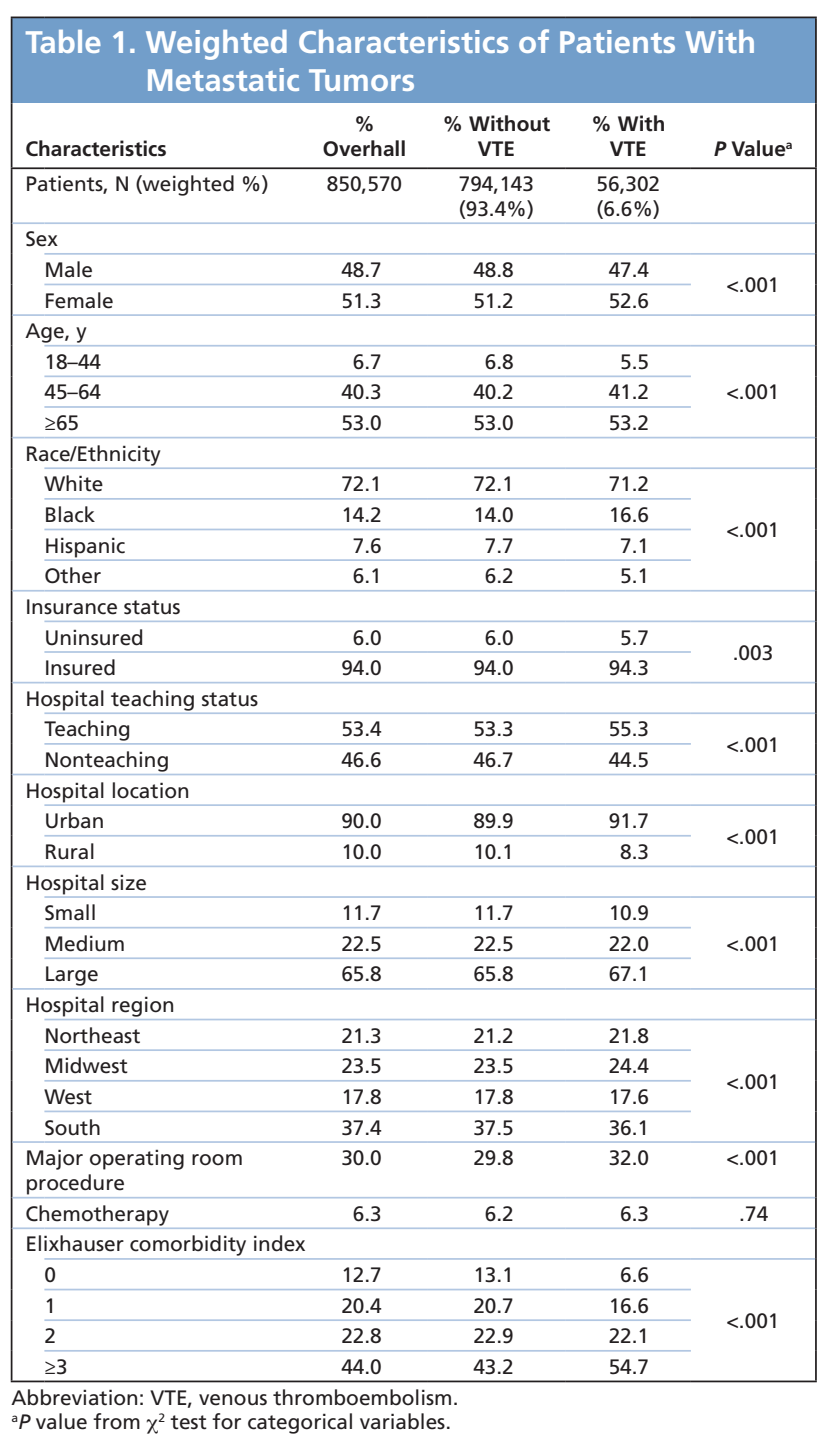

(26.9\%), lymph nodes (23.0\%), respiratory organs (19.5\%), and other gastrointestinal organs (13.3\%). Approximately $35.6 \%$ of the patients had multiple metastatic sites.

\section{Rates and Temporal Trends of VTE}

The overall prevalence of VTE was 6.6\%. VTE rates were not uniformly distributed across all subgroups of patients with metastatic cancer (Table 3). Primary cancer sites with the highest VTE rates included pancreas, uterus, cervix, ovary, lung, and stomach. The sites of tumor metastasis with the highest VTE rates included adrenal glands, liver, brain and spinal cord, and other gastrointestinal organs. A statistically significant trend for increasing VTE rates was 
VTE in Patients With Metastatic Cancer

\begin{tabular}{|c|c|c|c|c|}
\hline Tumor Characteristics & Overall \% & VTE \% & OR $(95 \% \mathrm{Cl})^{\mathrm{a}}$ & $P$ Value \\
\hline \multicolumn{5}{|l|}{ Primary tumor site ${ }^{b}$} \\
\hline Pancreas & 7.1 & 11.2 & $1.83(1.71-1.96)$ & $<.001$ \\
\hline Uterus & 2.3 & 9.4 & $1.44(1.33-1.57)$ & $<.001$ \\
\hline Cervix & 1.3 & 8.9 & $1.47(1.33-1.62)$ & $<.001$ \\
\hline Ovary & 5.0 & 7.7 & $1.25(1.16-1.35)$ & $<.001$ \\
\hline Lung \& bronchus & 29.2 & 7.5 & $1.14(1.07-1.22)$ & $<.001$ \\
\hline Stomach & 3.1 & 7.4 & $1.21(1.12-1.32)$ & $<.001$ \\
\hline Bladder & 2.7 & 7.2 & $1.12(1.03-1.21)$ & .005 \\
\hline $\begin{array}{l}\text { Gallbladder \& biliary } \\
\text { tract }\end{array}$ & 1.2 & 7.0 & $1.07(0.96-1.19)$ & .21 \\
\hline Testis & 0.6 & 6.9 & $1.29(1.07-1.55)$ & .008 \\
\hline Brain & 0.3 & 6.7 & $1.05(0.88-1.26)$ & .58 \\
\hline Esophagus & 2.1 & 6.5 & $1.01(0.92-1.10)$ & .85 \\
\hline Kidney \& renal pelvis & 3.9 & 5.8 & $0.77(0.71-0.84)$ & $<.001$ \\
\hline Liver & 2.1 & 5.6 & $0.83(0.76-0.91)$ & $<.001$ \\
\hline Colon/Rectum & 16.2 & 5.3 & $0.81(0.76-0.87)$ & $<.001$ \\
\hline Prostate & 7.8 & 5.3 & $0.82(0.76-0.88)$ & $<.001$ \\
\hline Bone & 0.7 & 5.2 & $0.91(0.78-1.06)$ & .23 \\
\hline Breast & 11.8 & 4.8 & $0.74(0.69-0.79)$ & $<.001$ \\
\hline Head \& neck & 3.5 & 2.8 & $0.44(0.40-0.49)$ & $<.001$ \\
\hline Thyroid & 1.3 & 2.4 & $0.41(0.35-0.48)$ & $<.001$ \\
\hline \multicolumn{5}{|l|}{ Metastatic site $^{b}$} \\
\hline Adrenal glands & 3.8 & 8.6 & $1.18(1.12-1.23)$ & $<.001$ \\
\hline Liver & 28.4 & 7.8 & $1.16(1.13-1.20)$ & $<.001$ \\
\hline Respiratory organs & 19.5 & 7.7 & $1.29(1.25-1.33)$ & $<.001$ \\
\hline $\begin{array}{l}\text { Gastrointestinal organs } \\
\text { (other than liver) }\end{array}$ & 13.3 & 7.5 & $1.03(0.99-1.07)$ & .113 \\
\hline Brain \& spinal cord & 14.8 & 7.4 & $1.14(1.10-1.18)$ & $<.001$ \\
\hline Other organs & 9.4 & 7.0 & $1.11(1.07-1.15)$ & $<.001$ \\
\hline Bone \& bone marrow & 26.9 & 7.0 & $1.18(1.15-1.22)$ & $<.001$ \\
\hline Unspecified site & 7.4 & 6.7 & $1.23(1.18-1.29)$ & $<.001$ \\
\hline Urinary organs & 2.4 & 6.6 & $0.97(0.90-1.04)$ & .36 \\
\hline Genital organs & 2.1 & 5.8 & $0.78(0.72-0.84)$ & $<.001$ \\
\hline Lymph nodes & 23.0 & 5.5 & $0.89(0.86-0.92)$ & $<.001$ \\
\hline \multicolumn{5}{|c|}{ Number of metastatic sites } \\
\hline Single & 64.4 & 6.0 & 1.00 [Ref] & \\
\hline Multiple ( $\geq 2$ ) & 35.6 & 7.7 & $1.09(1.05-1.13)$ & $<.001$ \\
\hline
\end{tabular}

Abbreviations: OR, odds ratio; VTE, venous thromboembolism. a'Model was weighted and adjusted for patients' clinical and sociodemographic characteristics, tumor characteristics, and hospital-level factors.

${ }^{b}$ Compared with all other cancers.

observed from 2008 to $2013(5.7 \%-7.2 \% ; P<.001)$ (Figure 1).

\section{Patient- and Hospital-Level Correlates of VTE}

Table 3 presents findings from the weighted adjusted multilevel analysis of patient- and hospital-level factors associated with the development of VTE in hospitalized patients with metastatic cancer. Higher odds of VTE were associated with female sex (odds ratio [OR], 1.04; 95\% CI, 1.02-1.06), black race (vs white; OR, 1.14; 95\% CI, 1.11-1.18), having a major operating room procedure (OR, 1.42; 95\% CI, 1.37$1.48)$, receipt of chemotherapy (OR, 1.08; $95 \% \mathrm{CI}$,
1.03-1.14), and Elixhauser comorbidity index score of $\geq 3$ (OR, 2.50; 95\% CI, 2.38-2.63). Hospital-level factors associated with higher odds of VTE were admission to a teaching hospital (OR, 1.05; 95\% CI, 1.01-1.11), urban location (OR, 1.18; 95\% CI, 1.09-1.27), and hospitals located in the Northeast (OR, 1.16; 95\% CI, 1.08-1.24) and West (OR, 1.09; 95\% CI, 1.03-1.16) compared with patients admitted to southern hospitals.

\section{Association of Primary and Secondary Tumor Site With VTE}

Table 2 presents the results of weighted adjusted multilevel regression analysis examining the association between primary and secondary site of the tumor and VTE. After controlling for the effect of patientand hospital-level factors, patients with metastatic cancer with primary tumors in the pancreas, uterus, cervix, ovary, lung, stomach, bladder, and testis had higher odds of VTE, whereas those with primary tumors in the breast, liver, prostate, kidney, colon/ rectum, head and neck, and thyroid had lower odds of VTE. Similarly, patients with tumor metastases to the adrenal glands, liver, brain and spinal cord, respiratory organs, and bone and bone marrow had higher odds of VTE whereas those with metastasis to lymph nodes and genital organs had lower odds of VTE. Having multiple $(\geq 2)$ metastatic sites increased the odds of VTE by 9\% (OR, 1.09; 95\% CI, 1.05-1.13) compared with a single metastatic tumor.

\section{Mortality and Hospital LOS After VTE}

Across all years in the study, the overall in-hospital mortality rate in patients with VTE was $12.4 \%$ compared with only $8.6 \%$ in patients without VTE (Table 4). In contrast to trends in VTE rates, a declining annual rate of in-hospital mortality was observed regardless of VTE diagnosis $(P<.001)$ (Figure $1)$. After adjusting for covariates in the multilevel regression analysis (Table 4), hospitalized patients with metastatic cancer diagnosed with VTE had 50\% (OR, 1.50; 95\% CI, 1.38-1.63) higher odds of in-hospital mortality relative to patients without VTE $(P<.001)$. Similarly, those with VTE had $65 \%$ (OR, 1.65; 95\% CI, 1.57-1.73) higher odds of experiencing longer hospital stays relative to those without VTE $(P<.001)$. 
Mohammed et al

\begin{tabular}{|c|c|c|c|}
\hline Characteristics & VTE \% & OR $(95 \%$ Cl) & $P$ Value \\
\hline Overall & 6.6 & - & - \\
\hline \multicolumn{4}{|l|}{ Sex } \\
\hline Female & 6.8 & $1.04(1.02-1.06)$ & $<.001$ \\
\hline Male & 6.4 & $1.00[$ Ref $]$ & \\
\hline \multicolumn{4}{|l|}{ Age, y } \\
\hline $18-44$ & 5.4 & $1.04(0.96-1.10)$ & 0.093 \\
\hline $45-64$ & 6.4 & $1.06(0.99-1.12)$ & 0.072 \\
\hline$\geq 65$ & 6.8 & $1.00[$ Ref $]$ & \\
\hline \multicolumn{4}{|l|}{ Race/Ethnicity } \\
\hline Black & 7.8 & $1.14(1.11-1.18)$ & $<.001$ \\
\hline Hispanic & 6.2 & $0.91(0.87-0.95)$ & $<.001$ \\
\hline Other & 5.5 & $0.83(0.79-0.87)$ & $<.001$ \\
\hline White & 6.6 & $1.00[$ Ref] & \\
\hline \multicolumn{4}{|l|}{ Insurance status } \\
\hline Uninsured & 6.3 & $0.97(0.93-1.02)$ & .21 \\
\hline Insured & 6.6 & $1.00[$ Ref] & \\
\hline \multicolumn{4}{|l|}{ Hospital teaching status } \\
\hline Teaching & 6.9 & $1.05(1.01-1.11)$ & .04 \\
\hline Nonteaching & 6.3 & $1.00[$ Ref $]$ & \\
\hline \multicolumn{4}{|l|}{ Hospital location } \\
\hline Urban & 6.7 & $1.18(1.09-1.27)$ & $<.001$ \\
\hline Rural & 5.4 & $1.00[$ Ref] & \\
\hline \multicolumn{4}{|l|}{ Hospital bed size } \\
\hline Small & 6.2 & $0.94(0.88-1.01)$ & .09 \\
\hline Medium & 6.5 & $0.94(0.90-1.00)$ & .04 \\
\hline Large & 6.7 & $1.00[$ Ref $]$ & \\
\hline \multicolumn{4}{|l|}{ Hospital region } \\
\hline Northeast & 6.8 & $1.16(1.08-1.24)$ & $<.001$ \\
\hline Midwest & 6.9 & $1.08(0.99-1.17)$ & .07 \\
\hline West & 6.6 & $1.09(1.03-1.16)$ & .002 \\
\hline South & 6.8 & $1.00[$ Ref] & \\
\hline Major operating room procedure & 7.1 & $1.42(1.37-1.48)$ & $<.001$ \\
\hline Chemotherapy & 6.6 & $1.08(1.03-1.14)$ & $<.001$ \\
\hline \multicolumn{4}{|l|}{ Elixhauser comorbidity index } \\
\hline 0 & 3.4 & $1.00[$ Ref] & \\
\hline 1 & 5.4 & $1.59(1.52-1.67)$ & $<.001$ \\
\hline 2 & 6.4 & $1.89(1.81-1.98)$ & $<.001$ \\
\hline$\geq 3$ & 8.2 & $2.50(2.38-2.63)$ & $<.001$ \\
\hline
\end{tabular}

Abbreviations: OR, odds ratio; VTE, venous thromboembolism.

aModel was weighted and adjusted for patients' clinical and sociodemographic characteristics, tumor characteristics, and hospital-level factors. Model was also adjusted for site of primary tumor, site of metastatic tumor, and receipt of chemotherapy.

\section{Discussion}

This study, involving 56,302 patients with VTE, is the largest study to date to determine the rates, correlates, and trends for VTE in hospitalized patients with metastatic cancer. Approximately, 6.6\% of patients with metastatic cancer in our analysis experienced VTE during hospitalization. This is higher than the rates reported by previous studies of hospitalized patients with cancer irrespective of their metastatic status; the previously reported range was $2.0 \%$ to $4.1 \%, 5,17$ indi-

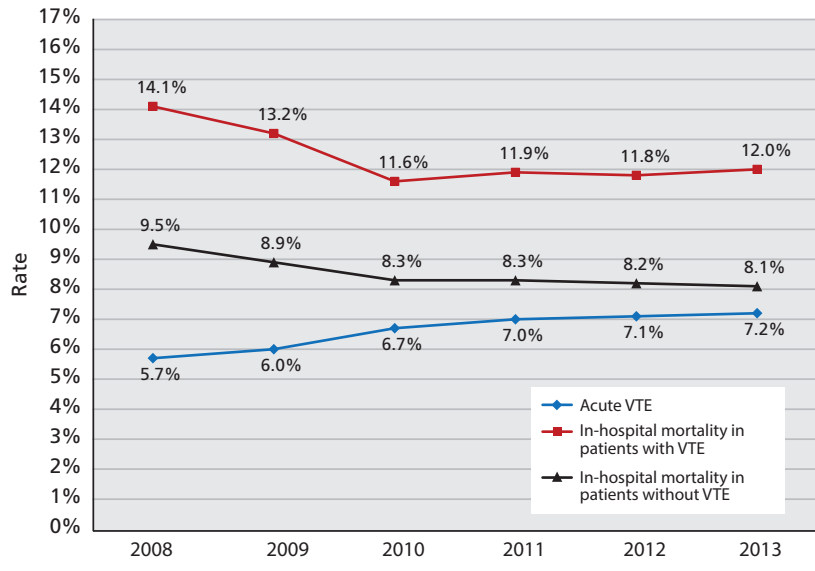

Figure 1. Rates of venous thromboembolism (VTE) among patients with metastatic cancer and overall in-hospital mortality among those with and without VTE between 2008 and 2013.

cating the greater vulnerability of patients with metastatic cancer to the development of VTE compared with those without metastasis.

Our analysis indicates a significant increase in VTE rates over the study period, which is consistent with previous studies. ${ }^{5,17}$ On the contrary, in-hospital mortality decreased in our study, despite the known association of inpatient mortality with VTE, which was previously estimated to increase mortality 2.5 times. ${ }^{5}$ It is possible that the increasing rate of VTE may be attributable to improvements in survival that result in more hospitalizations of patients with cancer. The trend of increased VTE incidence in hospitalized patients may also be the result of improved detection and increased surveillance. The phenomenon of surveillance bias in VTE is well described in trauma ${ }^{27}$ and surgical patients. ${ }^{10,12}$ Another reason for the increasing VTE trend among patients with cancer is the incidental finding of asymptomatic PE identified during CT scanning performed for other reasons, such as staging. ${ }^{28}$ Increased use of ultrasonography ${ }^{29}$ and $\mathrm{CT}^{30}$ over time confounds temporal analysis of VTE incidence and mortality. ${ }^{31}$ Increased surveillance and improved sensitivity of methods to detect VTE may lead to the diagnosis of VTE at an earlier stage, introducing both lead time and length bias in the assessment of mortality from VTE. Additionally, these findings reiterate the perception regarding greater clinician awareness of the association between VTE and cancer, and the increasing adoption of guidelines and evidence-based treatment and prophylaxis. ${ }^{32}$ 
VTE in Patients With Metastatic Cancer

Table 4. Association Between VTE and In-Hospital Mortality and Prolonged Hospitalization

\begin{tabular}{|lcccc|}
\hline Outcome Variable & No VTE, \% & VTE, \% & OR $(95 \% \text { Cl })^{\text {b }}$ & $P$ Value \\
\hline In-hospital mortality & 8.6 & 12.4 & $1.50(1.38-1.63)$ & $<.001$ \\
\hline Prolonged hospital stay & 27.0 & 40.7 & $1.65(1.57-1.73)$ & $<.001$ \\
\hline
\end{tabular}

Abbreviations: OR, odds ratio; VTE, venous thromboembolism. a'Weighted rate.

'Models were weighted and adjusted for patients' clinical and sociodemographic characteristics, tumor characteristics, hospital-level factors, and data collection year.

There were several notable patient-level characteristics associated with VTE in those with metastatic cancer. Consistent with findings of previous studies, ${ }^{5,6}$ higher odds of VTE were observed in women and patients with more comorbidities. In contrast to findings of previous studies, age was not a significant predictor for VTE in patients with metastatic cancer. ${ }^{5,6}$ A possible explanation could be that the biological aggressiveness of cancer metastasis predisposes all adult inpatients to VTE regardless of their age, especially after holding the effect of comorbidities constant. A significant increase in the odds of VTE was noted among black patients relative to white patients. Black patients have consistently been shown to be at increased risk of VTE, ${ }^{33}$ which has been attributed to the genetic variability in coagulation factors, particularly elevated factor VIII levels. ${ }^{34,35}$ Other studies have reported a lower use of thromboprophylaxis in black patients. ${ }^{36}$

Several hospital-level characteristics were found to be associated with VTE in patients with metastatic cancer. Admission to a teaching and an urban hospital significantly predicted the occurrence of VTE relative to nonteaching and rural hospitals. A possible explanation could be that cancer care centers are mostly based in urban and teaching hospitals, and that more complex patients are consistently referred to specialized centers for more advanced care. ${ }^{37}$ Furthermore, regional variation in the occurrence of VTE was evident in this analysis. Compared with the South, patients admitted to hospitals in Northeast and West had increased odds of developing VTE. This might suggest the difference in the implementation of thromboprophylaxis guidelines across the hospitals in different regions or differences in surveillance.

Consistent with previous studies, primary tumor sites associated with higher odds of VTE included pancreas, ovary, uterine, cervix, lung, stomach, and bladder. ${ }^{2,5,17}$ Moreover, patients with metastasis to liver, adrenal gland, brain, bone, and respiratory organs had higher odds of developing VTE, whereas those with metastasis to lymph nodes and genital organs had lower odds, even after controlling for the number of metastatic sites. Previous studies have documented the strong association between metastatic or regional-stage cancer and the development of VTE, specifically, in the first few months after diagnosis. ${ }^{2}$ To our knowledge, the current study is the first to report the independent association between specific metastatic sites of the tumor and VTE while adjusting for the effect of primary tumor type, patient sociodemographic variables, hospital characteristics, comorbidities, and other relevant covariates. It is possible that, in addition to primary tumor-specific prothrombotic mechanisms, cancer metastasis to certain organs, such as liver, lung, and adrenal glands, may be biologically more aggressive, resulting in increased hypercoagulability and a higher rate of VTE.

Patients with metastatic cancer with VTE had $48 \%$ higher odds of in-hospital mortality compared with those without VTE, which is consistent with findings of previous studies, but lower than previously reported..$^{5}$ Furthermore, patients with VTE had $63 \%$ higher odds of prolonged hospital stay relative to those without VTE, which is significantly longer than the hospitalization duration reported in previous studies, regardless of cancer stage and metastatic status. ${ }^{6}$ These findings might indicate the greater vulnerability of patients with metastatic cancer to the complications associated with VTE, and subsequently the negative impact VTE exerts on shortterm hospitalization outcomes. However, severity of illness and duration of hospitalization are associated with VTE, ${ }^{38,39}$ which confounds assessment of VTE complication. Given that some VTEs are preventable, there may be a need for stricter compliance with available prevention methods, such as thromboprophylaxis in high-risk patient populations, including those with metastatic cancer.

The strengths of this analyses include the large sample size used for powered analysis; the use of multilevel hierarchical regression to account for the patient-, tumor-, and hospital-level variation in the development of VTE; and the focus on patients with metastatic cancer, minimizing the need to account for the stage and grade of tumor, which the NIS data does not provide. 
This study has several limitations. First, our cross-sectional study design does not allow for evaluation of the directionality of the association to make any causal inference. Therefore, it is not possible to determine whether VTE occurred before admission or during hospitalization. Second, the NIS does not distinguish patients with multiple admissions, because it is based on unique admission rather than unique patient. Therefore, it is possible that repeated admissions of the same patients were included, and thereby VTE rates were underestimated or overestimated. Third, NIS data lacks information regarding medications; hence, we could not account for the use of VTE prophylaxis and treatment that might have influenced mortality rates and hospital LOS across different hospital settings. Third, administrative data have the potential to both overdiagnose and underdiagnose VTE cases, and are subject to coding errors. ${ }^{40,41}$ Furthermore, use of ICD-9 codes to identify the site of metastatic cancer for research purposes remains a challenge and a limitation associated with the use of NIS data, because studies have indicated that claims data might miss a significant percentage of cases based on the site of metastasis. ${ }^{42,43}$ Future studies should ascertain metastatic status through access to individual medical records or other confirmatory methods. Finally, the generalizability of these findings are confined to hospitalized patients with metastatic cancer in the United States.

\section{Conclusions}

This study highlights significant patient-, tumor-, and hospital-level attributes that are associated with the occurrence of VTE in patients with metastatic cancer. We found that VTE was strongly associated with in-hospital mortality and longer hospital stays. Although our study prevents providing clear guidelines on inpatient VTE, our findings corroborate those of previous studies aimed at identifying potential subgroups of patients who might be considered for thromboprophylaxis based on their tumor, sociodemographic, and other clinical characteristics. Particular attention can be given to patients with a primary tumor of lung and pancreas given the elevated risk and frequency of hospitalizations observed.

\section{References}

1. Sorensen HT, Mellemkjaer L, Olsen JH, Baron JA. Prognosis of cancers associated with venous thromboembolism. N Engl J Med 2000;343:18461850 .

2. Chew HK, Wun T, Harvey D, et al. Incidence of venous thromboembolism and its effect on survival among patients with common cancers. Arch Intern Med 2006;166:458-464.

3. Prandoni $\mathrm{P}$, Lensing AW, Cogo A, et al. The long-term clinical course of acute deep venous thrombosis. Ann Intern Med 1996;125:1-7.

4. Carson JL, Kelley MA, Duff A, et al. The clinical course of pulmonary embolism. N Engl J Med 1992;326:1240-1245.

5. Khorana AA, Francis CW, Culakova E, et al. Frequency, risk factors, and trends for venous thromboembolism among hospitalized cancer patients. Cancer 2007;110:2339-2346.

6. Streiff MB. Association between cancer types, cancer treatments, and venous thromboembolism in medical oncology patients. Clin Adv Hematol Oncol 2013;11:349-357.

7. Grosse SD, Nelson RE, Nyarko KA, et al. The economic burden of incident venous thromboembolism in the United States: a review of estimated attributable healthcare costs. Thromb Res 2016;137:3-10.

8. Levitan N, Dowlati A, Remick SC, et al. Rates of initial and recurrent thromboembolic disease among patients with malignancy versus those without malignancy. Risk analysis using Medicare claims data. Medicine (Baltimore) 1999;78:285-291.

9. Sallah S, Wan JY, Nguyen NP. Venous thrombosis in patients with solid tumors: determination of frequency and characteristics. Thromb Haemost 2002;87:575-579.

10. Holcomb CN, DeRussy A, Richman JS, Hawn MT. Association between inpatient surveillance and venous thromboembolism rates after hospital discharge. JAMA Surg 2015;150:520-527.

11. Trinh VQ, Karakiewicz PI, Sammon J, et al. Venous thromboembolism after major cancer surgery: temporal trends and patterns of care. JAMA Surg 2014;149:43-49.

12. Bilimoria KY, Chung J, Ju MH, et al. Evaluation of surveillance bias and the validity of the venous thromboembolism quality measure. JAMA 2013;310:1482-1489.

13. Haut ER, Pronovost PJ. Surveillance bias in outcomes reporting. JAMA 2011;305:2462-2463

14. Sousou $T$, Khorana A. Identifying cancer patients at risk for venous thromboembolism. Hamostaseologie 2009;29:121-124.

15. Agnelli G, Bolis G, Capussotti L, et al. A clinical outcome-based prospective study on venous thromboembolism after cancer surgery: the @ RISTOS project. Ann Surg 2006;243:89-95.

16. Khorana AA, Francis CW, Culakova E, Lyman GH. Risk factors for chemotherapy-associated venous thromboembolism in a prospective observational study. Cancer 2005;104:2822-2829.

17. Stein PD, Beemath A, Meyers FA, et al. Incidence of venous thromboembolism in patients hospitalized with cancer. Am J Med 2006;119:60-68.

18. Beaton R, Pagdin-Friesen W, Robertson C, et al. Effects of exercise intervention on persons with metastatic cancer: a systematic review. Physiother Can 2009;61:141-153.

19. Caine GJ, Stonelake PS, Lip GY, Kehoe ST. The hypercoagulable state of malignancy: pathogenesis and current debate. Neoplasia 2002;4:465-473.

20. Healthcare Cost and Utilization Project (HCUP). Overview of the National (Nationwide) Inpatient Sample (NIS). Available at: https:/ www.hcup-us.ahrq.gov/nisoverview.jsp. Accessed January 3, 2018.

21. Perioperative Pulmonary Embolism or Deep Vein Thrombosis Rate: Technical Specifications. Available at: https://www.qualityindicators. ahrq.gov/Downloads/Modules/PSI/V45/TechSpecs/PSI\%2012\%20 Perioperative\%20Pulmonary\%20Embolism\%20or\%20Deep\%20Vein\%20 Thrombosis\%20Rate.pdf. Accessed July 25, 2017.

22. Tamariz L, Harkins T, Nair V. A systematic review of validated methods for identifying venous thromboembolism using administrative and claims data. Pharmacoepidemiol Drug Saf 2012;21(Suppl 1):154-162.

23. Sukumar $S$, Roghmann F, Trinh VQ, et al. National trends in hospitalacquired preventable adverse events after major cancer surgery in the USA. BMJ Open 2013;3:pii: e002843. 
24. Elixhauser A, Steiner C, Harris DR, Coffey RM. Comorbidity measures for use with administrative data. Med Care 1998;36:8-27.

25. Kakkar AK, Haas S, Wolf H, Encke A. Evaluation of perioperative fatal pulmonary embolism and death in cancer surgical patients: the MC-4 cancer substudy. Thromb Haemost 2005;94:867-871

26. Kakkar AK. Prevention of venous thromboembolism in the cancer surgical patient. J Clin Oncol 2009;27:4881-4884.

27. Pierce CA, Haut ER, Kardooni S, et al. Surveillance bias and deep vein thrombosis in the national trauma data bank: the more we look, the more we find. J Trauma 2008;64:932-936; discussion 936-937.

28. Khorana AA, O'Connell C, Agnelli G, et al. Incidental venous thromboembolism in oncology patients. J Thromb Haemost 2012;10:26022604.

29. Stein PD, Hull RD, Ghali WA, et al. Tracking the uptake of evidence: two decades of hospital practice trends for diagnosing deep vein thrombosis and pulmonary embolism. Arch Intern Med 2003;163:1213-1219.

30. DeMonaco NA, Dang Q, Kapoor WN, Ragni MV. Pulmonary embolism incidence is increasing with use of spiral computed tomography. Am J Med 2008;121:611-617.

31. Burge AJ, Freeman KD, Klapper PJ, Haramati LB. Increased diagnosis of pulmonary embolism without a corresponding decline in mortality during the CT era. Clin Radiol 2008;63:381-386.

32. Qaseem A, Snow V, Barry P, et al. Current diagnosis of venous thromboembolism in primary care: a clinical practice guideline from the American Academy of Family Physicians and the American College of Physicians. Ann Fam Med 2007;5:57-62.

33. Heit JA, Beckman MG, Bockenstedt PL, et al. Comparison of characteristics from White- and Black-Americans with venous thromboembolism: a cross-sectional study. Am J Hematol 2010;85:467-471.
34. Austin H, De Staercke C, Lally C, et al. New gene variants associated with venous thrombosis: a replication study in white and black Americans. J Thromb Haemost 2011;9:489-495.

35. Patel RK, Ford E, Thumpston J, Arya R. Risk factors for venous thrombosis in the black population. Thromb Haemost 2003;90:835-838.

36. McGarry LJ, Thompson D. Retrospective database analysis of the prevention of venous thromboembolism with low-molecular-weight heparin in acutely III medical inpatients in community practice. Clin Ther 2004;26:419-430.

37. Lave JR, Lave LB. The extent of role differentiation among hospitals. Health Serv Res 1971;6:15-38.

38. Spyropoulos AC, Anderson FA Jr, FitzGerald G, et al. Predictive and associative models to identify hospitalized medical patients at risk for VTE. Chest 2011;140:706-714.

39. Amin AN, Varker H, Princic N, et al. Duration of venous thromboembolism risk across a continuum in medically ill hospitalized patients. J Hosp Med 2012;7:231-238

40. Lawthers AG, McCarthy EP, Davis RB, et al. Identification of in-hospital complications from claims data. Is it valid? Med Care 2000;38:785-795.

41. White RH, Garcia M, Sadeghi B, et al. Evaluation of the predictive value of ICD-9-CM coded administrative data for venous thromboembolism in the United States. Thromb Res 2010;126:61-67.

42. Liede $A$, Hernandez RK, Roth $M$, et al. Validation of International Classification of Diseases coding for bone metastases in electronic health records using technology-enabled abstraction. Clin Epidemiol 2015;7:441448.

43. Nordstrom BL, Whyte JL, Stolar M, et al. Identification of metastatic cancer in claims data. Pharmacoepidemiol Drug Saf 2012;21(Suppl 2):2128.

See JNCCN.org for supplemental online content. 